\title{
Sistema de razonamiento basado en casos, para la mejora de atención
} de salud en un centro rural

Case based reasoning system for the improvement of health care in a rural center

Miguel Angel Aguilar Luna Victoria', Augusto Cortéz Vásquez²

\section{RESUMEN}

Objetivo: Crear un modelo de sistema de razonamiento basado en casos como apoyo al médico en el diagnóstico, para la mejora de la atención de salud de un poblador en un centro rural. Métodos: La población en estudio estuvo constituida por los asistentes a las postas médicas de la Comunidad Campesina Cruz de Mayo, tomándose una muestra de 40 casos. La investigación estuvo enmarcada dentro del desarrollo tecnológico, orientada a la obtención de un modelo de sistema de razonamiento basado en casos, que no depende únicamente de conocimiento general del dominio del problema, sino que utilizó los diagnósticos de casos pasados, almacenados en una base de casos. El enfoque metodológico utilizado fue el descriptivo-analítico, no experimental, además de cuantitativo al cuantificar las puntuaciones de acierto que el modelo proporciona, para lo cual se construyó la estructura de casos sobre los síntomas más comunes de dos enfermedades: malaria y leishmaniasis. Se usó los softwares JCOLIBRI y Protegé, para la construcción del sistema y la ontología dominio. Resultados: La implementación fue exitosa, probándola con las dos enfermedades en estudio, suficientes para demostrar que se ejecuta de acuerdo a los requerimientos de ingreso (síntomas), mostrando por pantalla los casos cuyos síntomas son idénticos a los ingresados. Conclusiones: La concordancia de diagnóstico para las enfermedades en mención, entre el modelo propuesto y el diagnóstico médico fue alta, resultó un índice de Kappa de Kohen de 0,89 ( $p<0,01)$, indicando que el acercamiento entre ambos diagnósticos es muy bueno dentro de la escala de valoración de este indicador.

Palabras clave: Sistema de razonamiento basado en casos (SRBC), similitud de casos, base de casos, conector, diagnóstico basado en casos, malaria, leishmaniasis

\section{ABSTRACT}

Objective: To create a model of reasoning system based on cases as support to the doctor in the diagnosis, for the improvement of the health care of an individual in a rural center. Methods: The study population consisted for inhabitants who attend in the medical post of Cruz de Mayo rural community, taking a sample of 40 cases. The research was framed within the technological development, oriented to obtain a model of reasoning system based on cases, that doesn't only depend on general knowledge of the problem, but it used the diagnoses of past cases, stored in a database cases. The methodological approach used was the descriptive-analytical, not experimental, and quantitative when quantifying the correct scores provided by the model, for which was built the case structure on the most common symptoms of two diseases: malaria and leishmaniasis. The software JCOLIBRI and Protegé were used, for the construction of the system and domain ontology. Results: The implementation was successful, proving it with the two diseases studied, sufficient to demonstrate that it is executed according to the requirements of admission (symptoms), showing by screen the cases whose symptoms are identical of those admitted. Conclusions: The concordance of diagnosis for the mentioned diseases, between the proposed model and the medical diagnosis was high, resulting a Kappa Kohen index of $0.89(p<0.01)$, indicating that the approach between both diagnoses is very good within the rating scale of this indicator.

Keywords: Case-based reasoning system (CBRS), similarity of cases, case-base, connector, casebased diagnosis, malaria, leishmaniasis. 


\section{INTRODUCCIÓN}

En nuestro país, en los lugares alejados de las capitales de provincia (sierra y selva), existe carencia de hospitales y especialistas, donde a los médicos les toca diagnosticar enfermedades y en otros casos ejecutar prácticas que en ocasiones no son de su especialidad. Es así que a menudo se presentan casos al médico y éste quiere conocer cómo respondieron otros pacientes al tratamiento; sin embargo, no cuenta con un repositorio de casos ni de sus tratamientos en el que se registre la experiencia de otros médicos acerca de tal o cual descripción de enfermedad, su tratamiento, los efectos, la duración de la enfermedad y otras tantas características, especialmente de casos encontrados en las lejanías de nuestro país para que le permita tomar una decisión acertada. Esto significa que el especialista al diagnosticar la enfermedad y al analizar los casos similares se informa mejor sobre los tratamientos dados a otros, de la misma manera al contrario, al indicarse los síntomas puede ayudar al médico a un diagnóstico más certero. Obviamente no todos los pacientes tendrán los mismos síntomas y es allí donde el sistema experto puede ser en el caso de la medicina de gran utilidad.

En el más remoto contexto rural, todo el cuidado debe ser asumido en un pequeño hospital o posta médica, por médicos, practicantes y enfermeras en un número reducido, donde inclusive no existen especialidades. Este personal de salud no solamente es responsable de emergencias médicas sino también de otros campos de la medicina incluyendo práctica de comunidad general y la administración misma del centro de salud.

El razonamiento basado en casos (RBC), cubre un amplio rango de métodos para generar, organizar, recuperar y utilizar el conocimiento retenido de casos pasados. El dominio de RBC es versátil y ha encontrado muchas aplicaciones en varios campos tales como la medicina, ingeniería, leyes, finanzas y manufactura; por decir unos pocos. Este sistema también ha sido satisfactoriamente aplicado para resolver problemas de clasificación de patrones encontrados frecuentemente en los campos antes mencionados, Aamodt y Plaza (1994).
Dicho lo anterior, esta investigación responde a la necesidad urgente de paliar las necesidades básicas de salud en los centros rurales alejados de nuestro país mediante el sistema de razonamiento basado en casos (RBC), ya que con éste se tendría un apoyo al médico como especialista en salud en cuanto al diagnóstico en el que se centra esta investigación, lo cual serviría no sólo para la solución de emergencias, sino inclusive, para realizar una acción preventiva de las enfermedades a favor de los pobladores alejados a muchos kilómetros de distancia de un verdadero centro poblado que tenga los recursos médicos.

En lo que respecta al campo de la medicina, se puede afirmar que esta metodología se ajusta muy bien al desempeño de los profesionales de salud, cuando se presenta un caso de enfermedad, incorporándolo dentro de su escenario clínico. Sobre todo, el RBC es atractivo en el entorno médico, porque un caso base ya existente tiene almacenado en sí mismo los síntomas, diagnóstico, tratamiento y resultado para cada paciente (Andritsos, Jurisica y Glasgow, 2014).

Existen varios antecedentes internacionales (mas no nacionales) para la aplicación de este sistema, en Cuba, por ejemplo, se tiene un sistema RBC en Ciencias Médicas Sobre Plataforma Web 8 (Delgado, 2009), cuyo valor práctico consiste en su carácter eminentemente docente y clínico, ya que facilita tanto a estudiantes de medicina y de enfermería, así como residentes y médicos especialistas a que puedan interactuar con Bases de Casos que permitan ayudarlos en el proceso del diagnóstico.

Por otro lado, en España, el Grupo de Aplicaciones de Inteligencia Artificial (GAIA) con (Recio-García, Díaz-Agudo y GonzálezCalero, 2014) de la Universidad Complutense, ha desarrollado la plataforma JColibrí, (software libre). Esta plataforma permite crear aplicaciones de RBC para la mayoría de las áreas del conocimiento, de forma rápida y sencilla, siendo utilizada en la actualidad como herramienta educativa, en más de una docena de universidades de todo el mundo. Cuenta además, con el apoyo de varios grupos internacionales de investigación que contribuyen a su desarrollo. 
El objetivo pues, de esta investigación es el de crear un modelo de sistema de razonamiento basado en casos como apoyo al médico en el diagnóstico, para la mejora de la atención de salud de un poblador en un centro rural.

\section{MATERIAL Y MÉTODOS}

Para nuestro estudio se escogió como población objetivo a los habitantes del ámbito rural de la Comunidad Campesina Cruz de Mayo, formada por 13 sectores y que según el último censo de población tiene 3700 habitantes dentro de una extensión de tierras comunes que superan las 4000 hectáreas. Se consultó con los médicos, cuál o cuáles eran las enfermedades localizadas en esta región, llegando a la conclusión que la malaria y leishmaniasis, aunque no están en un porcentaje extremo, pues sólo afecta al $1 \%$ y $0,1 \%$ de la población respectivamente; sin embargo, por el mismo hecho que su sintomatología es muy penosa y puede conllevar a la muerte, desearían exterminarlas totalmente.

La muestra recolectada, fue a través de un muestreo no probabilístico por conveniencia, en la que se tomó como referencia un tipo de enfermedad (malaria y posteriormente leishmaniasis), escogiéndose a los sujetos en estudio que padecen la misma. Se tomaron la totalidad de casos: 40 casos de malaria, 5 de leishmaniasis; y por motivos de ilustrar esta investigación, cargamos además 5 pacientes indicados como sanos en su historial médico. Para la toma de información se tomó como marco muestral los padrones de historias clínicas de la Comunidad Campesina Cruz de Mayo.

Para esta investigación se utilizó el software Protégé [Protégé, 2014], el cual es un editor de ontologías de código abierto y un sistema de adquisición de conocimiento, desarrollado por la Universidad de Stanford, en colaboración con la Universidad de Manchester; y por otro lado el software jColibri, entorno de desarrollo orientado a la creación de sistemas de razonamiento basado en casos. es un producto del Grupo de Aplicaciones de Inteligencia Artificial, conocido por sus siglas GAIA, grupo integrado por profesores y estudiantes (RecioGarcía, Díaz-Agudo y González-Calero, 2014) interesados en el campo de la Inteligencia Artificial de la Universidad Complutense de Madrid. A continuación su historia de este entorno como motivación al estudio.

\section{RESULTADOS}

Acorde a la metodología RBC, nuestro modelo de sistema se resume en los siguientes pasos.

-El sistema buscará en la memoria de casos un caso existente que se ajuste al problema ingresado.

El sistema encontrará un caso que coincide exactamente con el problema de entrada y va directamente a una solución (el sistema se hará más potente mientras se añade nuevos casos).

Si no encontrara un caso exactamente igual al planteado, se recupera un caso que es similar a nuestra situación de entrada, pero no del todo adecuada para proporcionar una solución completa (Cunningham, 2009).

- El sistema debe encontrar y modificar pequeñas porciones del caso de que no cumplan con la especificación de entrada recuperada. Este proceso se llama "casoadaptación".

-El resultado de proceso de adaptación a un caso es (a) completar la solución, y (b) generar un nuevo caso que se agrega automáticamente a la memoria del sistema para uso futuro.

Para esto, es necesario establecer los síntomas de ambas enfermedades los cuales se muestran en la Tabla. 
Tabla 1. Parámetros de evaluación en el diagnóstico de las enfermedades

\begin{tabular}{|c|c|c|c|}
\hline $\begin{array}{l}\text { Parámetros de } \\
\text { Evaluación }\end{array}$ & Niveles & $\begin{array}{l}\text { Parámetros de } \\
\text { Evaluación }\end{array}$ & Niveles \\
\hline Anemia & $\begin{array}{l}\text { Sin anemia } \\
\text { Baja } \\
\text { Media } \\
\text { Severa }\end{array}$ & Anemia & $\begin{array}{l}(2-4) \text { días } \\
(4-5) \text { días } \\
(5-7) \text { dias } \\
>7 \text { días }\end{array}$ \\
\hline Congestión nasal & $\begin{array}{l}\text { Sin congestión } \\
\text { Hemorragia nasal } \\
\text { Rinorrea }\end{array}$ & Heces con sangre & $\begin{array}{l}\text { Heces negras } \\
\text { Heces rojo obscuro }\end{array}$ \\
\hline Convulsión & $\begin{array}{l}\text { Sin convulsión } \\
\text { Desvanecimiento } \\
\text { breve } \\
\text { Estremeciniento } \\
\text { Espasmos }\end{array}$ & Hemorragia nasal & $\begin{array}{l}\text { Sin Hemorragia } \\
\text { Con Hemorragia }\end{array}$ \\
\hline Dificultad para deglutir & $\begin{array}{l}\text { Sin dificultad } \\
\text { Baja } \\
\text { Media } \\
\text { Severa }\end{array}$ & Hepatomegalia & $\begin{array}{l}\text { Higado normal } \\
\text { Hígado hinchado }\end{array}$ \\
\hline Dificultad para respirar & $\begin{array}{l}\text { Sin dificultad } \\
\text { Baja } \\
\text { Media } \\
\text { Severa }\end{array}$ & Ictericia & $\begin{array}{l}\text { Boca } \\
\text { Heces } \\
\text { Ojos y piel } \\
\text { Orina }\end{array}$ \\
\hline Dolor de cabeza & $\begin{array}{l}\text { Sin dolor } \\
\text { Con dolor }\end{array}$ & Náuseas & $\begin{array}{l}\text { Sin náuseas } \\
\text { Con náuseas }\end{array}$ \\
\hline Dolor muscular & $\begin{array}{l}\text { Sin dolor } \\
\text { Con dolor }\end{array}$ & Sudoración & $\begin{array}{l}\text { Sin sudor } \\
\text { Sudor frío }\end{array}$ \\
\hline Escalofrios & $\begin{array}{l}\text { Sin escalofrios } \\
\text { Con escalofrios }\end{array}$ & Temperatura & $\begin{array}{l}\text { Normal } \\
\leq 38 \\
(38-39) \\
(39.40) \\
\geq 40\end{array}$ \\
\hline Esplenomegalia & $\begin{array}{l}\text { Bazo normal } \\
\text { Bazo hinchado }\end{array}$ & Úlcera cutánea & $\begin{array}{l}\text { Úlcera en la boca } \\
\text { Úllcera en las encías } \\
\text { Úlcera en la piel } \\
\text { Úlcera en el tabique }\end{array}$ \\
\hline
\end{tabular}

Fuente: http://www.icd10data.com/ICD10CM/Codes/A00-B99/B50-B64/B54-/B54

La Figura 1 ilustra la ontología obtenida para nuestro estudio, mediante el software Protégé, conteniendo la descripción de clases, propiedades e instancias. En la Figura 2 se muestra un caso de un paciente en particular con sus síntomas correspondientes. En
Protégé, sólo se ha construido las clases y subclases, así como las propiedades de los objetos y las características individuales (niveles de los síntomas) para cada subclase y en la Figura 3 se muestra la asignación de la ontología a la estructura de caso. 


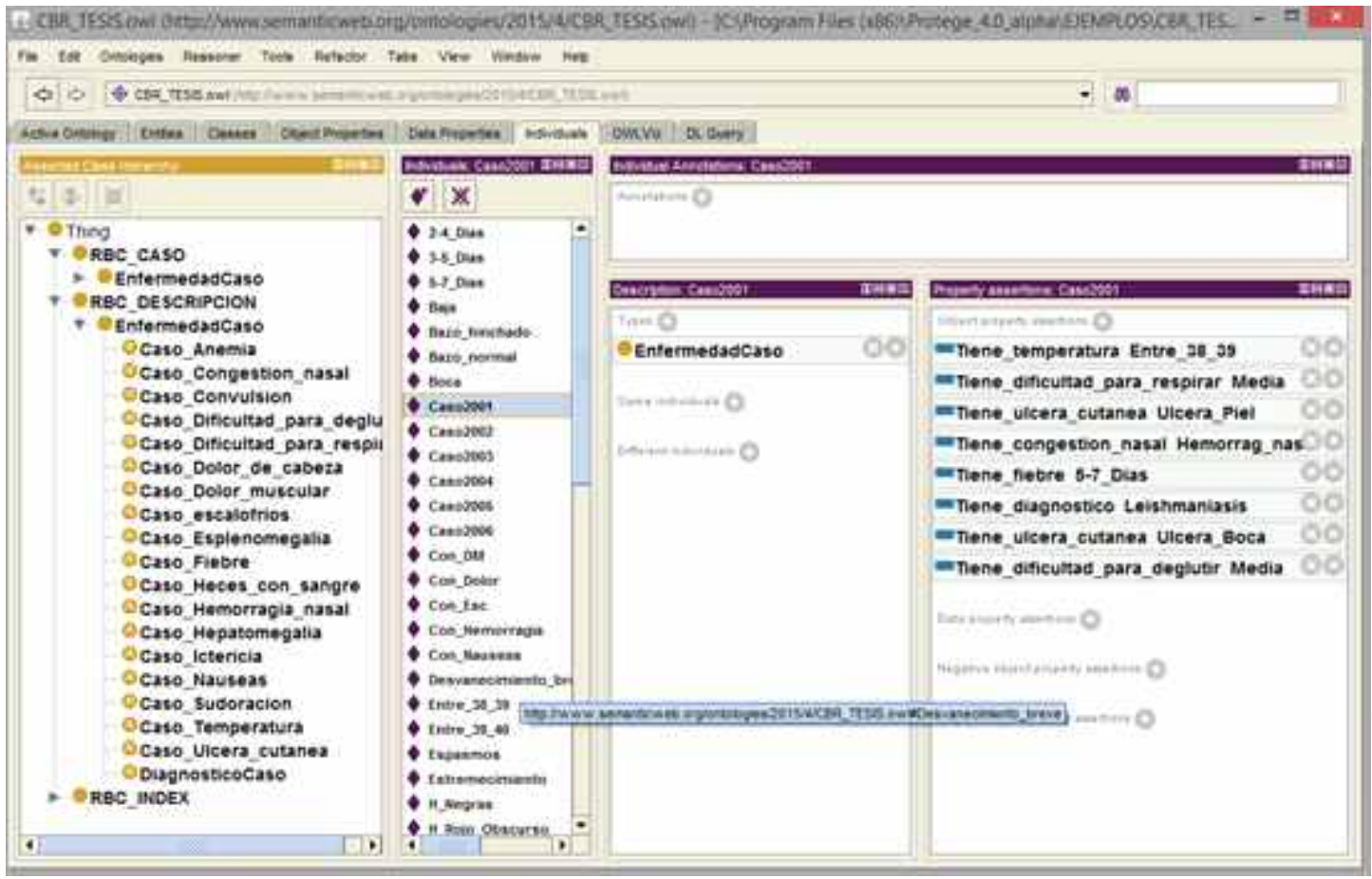

Figura 1. Creación de clases y relaciones en Software Protégé

\begin{tabular}{|c|c|c|}
\hline 4 & & $x$ \\
\hline 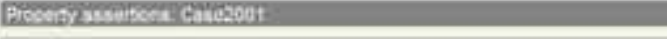 & \multicolumn{2}{|c|}{ 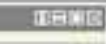 } \\
\hline \multicolumn{2}{|l|}{ Obieat piepenty sanitians 0} & 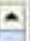 \\
\hline Enene_congestion_nasal Hemorrag_nasal & 30 & \\
\hline ETiene_hemorragia_nasal Con_Hemorragia & 80 & \\
\hline m Tiene_dolor_de_cabeza Sin_Dolor_Cabeza & Q6 & \\
\hline mene_ictericia Sin_Ictericia & 60 & \\
\hline \multicolumn{3}{|l|}{ =Tiene_dificultad_para_deglutir Dif_Degiutir_Media } \\
\hline \multicolumn{3}{|l|}{ Eliene_diagnostico Leishmaniasis } \\
\hline = Tene_hepatomegalia Higado_normal & 80 & \\
\hline \multicolumn{3}{|l|}{ mene_nauseas Con_Nauseas } \\
\hline = Tiene_temperatura Entre_38_39 & 60 & \\
\hline Niene_convulsion Espasmos & 60 & \\
\hline \multicolumn{3}{|l|}{ = Tene_anemia Anemia_Baja } \\
\hline \multicolumn{3}{|l|}{ = Tene_dificultad_para_respirar Dif_Respirar_Media } \\
\hline = Tiene_dolor_muscular Con_DM & 60 & \\
\hline \multicolumn{3}{|l|}{ = Tiene_ulcera_cutanea Uicera_Piel } \\
\hline Ninene_escalotrios Con_Esc & $\overline{60}$ & \\
\hline m Tene_sudoracion Sudor_Frio & 80 & \\
\hline \multicolumn{3}{|l|}{ = Tiene_espienomegalia Bazo_normal } \\
\hline mene_fiebre 5.7_Dias & 60 & \\
\hline = Tene_heces_con_sangre Normal & 80 & \\
\hline 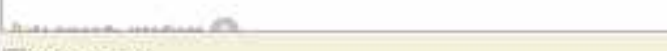 & & - \\
\hline U. Synchrosiang & & \\
\hline
\end{tabular}

Figura 2. Caso 2001 y sus síntomas, elaborado en PROTÉGÉ 


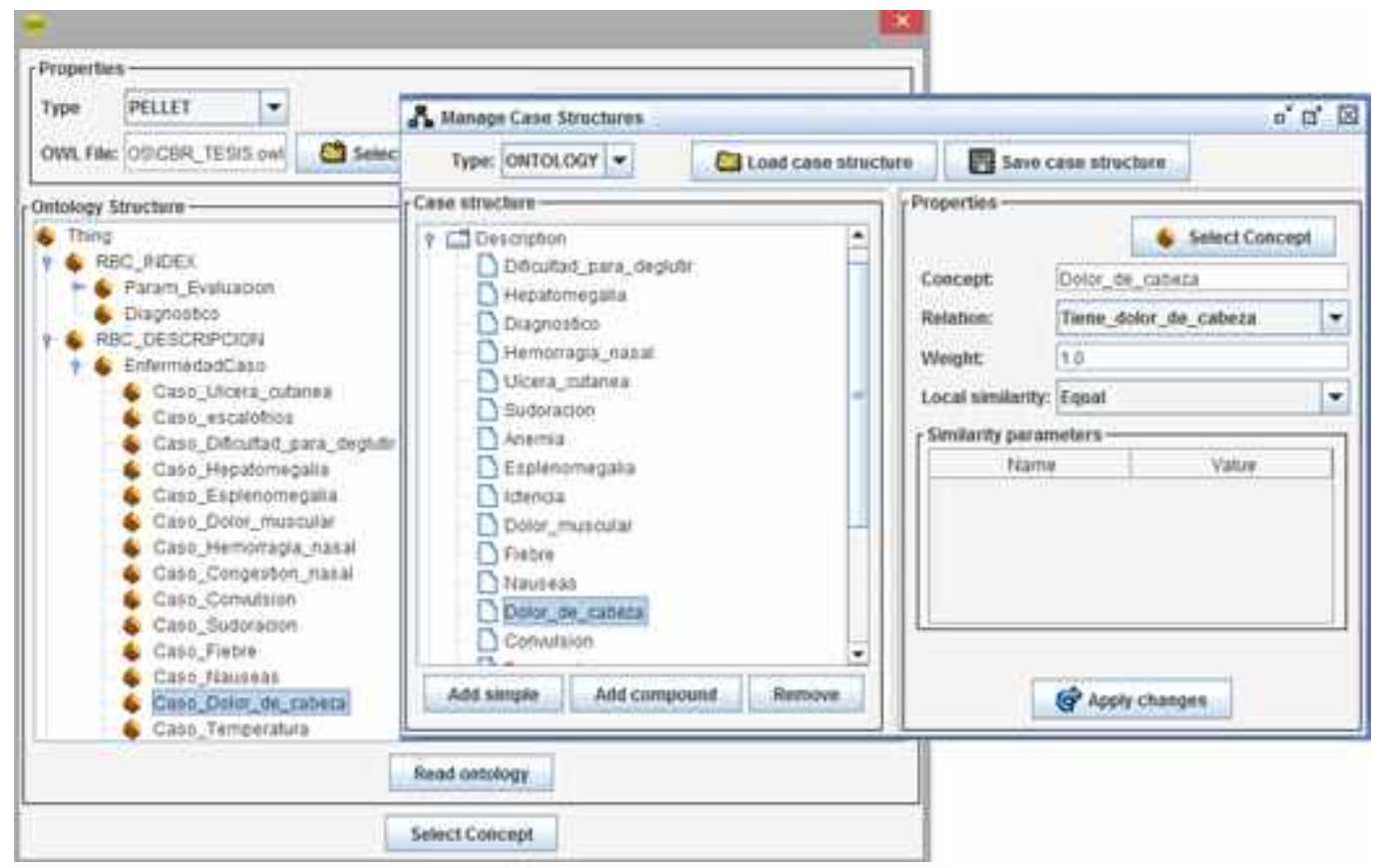

Figura 3. Asignación de la estructura del caso a la ontología en jCOLIBRI

\begin{tabular}{|c|c|c|c|c|}
\hline \multicolumn{4}{|c|}{ Query } & $x$ \\
\hline \multicolumn{5}{|l|}{ Requested parameters } \\
\hline Ulcera_cutanea & <empty> & $\checkmark$ & $\psi 0.0$ & 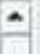 \\
\hline Sudoracion & Sudor_frio & $\nabla$ & $\Longrightarrow 1.0$ & \\
\hline Anemia & Media & $\checkmark$ & $\bigcup_{1.0}$ & \\
\hline Esplenomegalia & Bazo_hinchado & - & $\Longrightarrow 1.0$ & 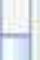 \\
\hline Ictericia & Ojos_Piel & $\checkmark$ & $\bigcup_{1.0}$ & \\
\hline Dolor_muscular & Con_DM & - & $\Longrightarrow / 1.0$ & \\
\hline Fiebre & Dias & 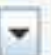 & $\longrightarrow 1.0$ & \\
\hline Dolor_de_cabeza & Con_Dolor & 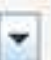 & $\Longrightarrow 1.0$ & \\
\hline Convulsion & Estremecimiento & $\checkmark$ & $\bigcup_{1.0}$ & $=$ \\
\hline Temperatura & Entre_38_39 & 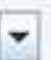 & $\longrightarrow \bigcup_{1.0}$ & \\
\hline Escalotrios & Con_Esc & - & $\Longrightarrow 1.0$ & \\
\hline Congestion_nasal & <empty> & - & 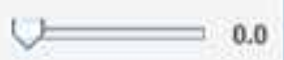 & \\
\hline Heces_con_sangre & H_Negras & $\nabla$ & $\bigcup_{1.0}$ & \\
\hline Dificultad_para_respirar & <empty & - & $\sqcup 0.0$ & 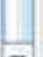 \\
\hline \multirow[t]{2}{*}{1} & \multicolumn{2}{|l|}{ III } & || & \\
\hline & Ok & & & \\
\hline
\end{tabular}

Figura 4. Pantalla de consulta a manejar por el especialista 
Finalmente la Figura 4 muestra la pantalla a manejar por el especialista. Nuestra aplicación RBC está compuesta por un Pre-Ciclo, el cual tiene la tarea de llamar los casos; el Ciclo, el cual tiene cuatro tareas fundamentales que son consulta, recuperación, reuso, revisión y captación; y, un Post-Ciclo que almacena los casos. Cada tarea puede ser desarrollada por varios métodos y que pueden ser escogidos por el usuario. Para nuestra investigación se utilizó dos métodos para resolver el problema:

Descomposición, divide la tarea principal en sub-tareas.

-Resolución, resuelve la tarea directamente.
Al lado derecho de cada parámetro, se tiene la ponderación de cada uno de ellos y que puede ser establecido por el especialista en salud, simplemente desplazando la barra deslizante, teniendo a 0 y 1 como los valores de menor y mayor peso.

Luego de alimentar la pantalla de consulta, el software está listo para procesar la información ingresada. La Figura 5 muestra los resultados de la tarea de recuperación, se puede observar que el caso más similar al consultado es el caso 2044 con una similitud del $89,47 \%$

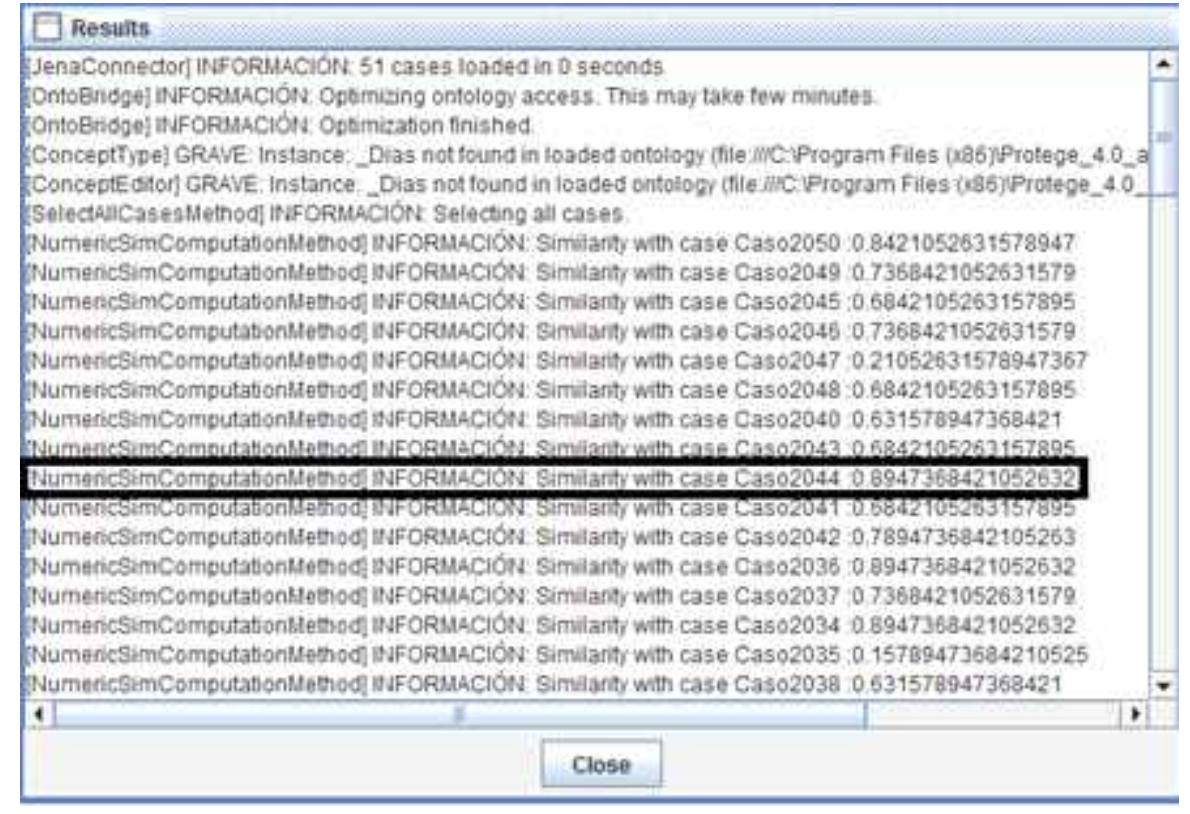

Figura 5. Pantalla de salida, mostrando las similitudes con el caso en consulta

Para medir la confiabilidad de los resultados obtenidos con nuestra aplicación RBC, se realizó un análisis descriptivo de los 50 casos en estudio (45 enfermos y 5 sanos) comparando el diagnóstico dado por el médico, escrito en los padrones de historias clínicas y el de la simulación dada por nuestro modelo ejecutado para los 45 casos de enfermedad, encontrándose los resultados mostrados en la Tabla 2, siendo que el porcentaje de acierto de diagnóstico para la enfermedad leishmaniasis es del $100 \%$, para el diagnóstico de la malaria fue del $98 \%$ y para el diagnóstico de sano es de $80 \%$.

Asimismo, se recurrió al análisis estadístico, con la finalidad de dar un sustento más científico y valedero al estudio y así comprobar el grado de concordancia entre el modelo RBC propuesto y el diagnóstico médico (obtenido de los archivos clínicos) para los 45 pacientes de la muestra. 
Tabla 2. Diagnóstico del experto y el modelo RBC propuesto

\begin{tabular}{|c|c|c|c|c|c|}
\hline $\begin{array}{c}\text { Caso } \\
\text { Evaluado }\end{array}$ & $\begin{array}{l}\text { Experto } \\
\text { (médico) }\end{array}$ & $\begin{array}{l}\text { Modelo } \\
\text { RBC }\end{array}$ & Caso Evaluado & Experto(médico) & Modelo RBC \\
\hline Caso_2001 & Leishmaniasis & Leishmaniasis & Caso_2026 & Leishmaniasis & Leishmaniasis \\
\hline Caso_2002 & Sano & Malaria & Caso_2027 & Leishmaniasis & Leishmaniasis \\
\hline Caso_2003 & Malaria & Malaria & Caso_2028 & Malaria & Malaria \\
\hline Caso_2004 & Leishmaniasis & Leishmaniasis & Caso_2029 & Malaria & Malaria \\
\hline Caso_2005 & Leishmaniasis & Leishmaniasis & Caso_2030 & Malaria & Malaria \\
\hline Caso_2006 & Malaria & Malaria & Caso_2031 & Malaria & Malaria \\
\hline Caso_2007 & Malaria & Malaria & Caso_2032 & Malaria & Malaria \\
\hline Caso_2008 & Malaria & Malaria & Caso_2033 & Malaria & Malaria \\
\hline Caso_2009 & Malaria & Malaria & Caso_2034 & Malaria & Malaria \\
\hline Caso_2010 & Malaria & Malaria & Caso_2035 & Sano & Sano \\
\hline Caso_2011 & Malaria & Malaria & Caso_2036 & Malaria & Malaria \\
\hline Caso_2012 & Malaria & Malaria & Caso_2037 & Malaria & Malaria \\
\hline Caso_2013 & Malaria & Malaria & Caso_2038 & Malaria & Malaria \\
\hline Caso_2014 & Malaria & Malaria & Caso_2039 & Sano & Sano \\
\hline Caso_2015 & Malaria & Malaria & Caso_2040 & Malaria & Malaria \\
\hline Caso_2016 & Malaria & Leishmaniasis & Caso_2041 & Malaria & Malaria \\
\hline Caso_2017 & Malaria & Malaria & Caso_2042 & Malaria & Malaria \\
\hline Caso_2018 & Malaria & Malaria & Caso_2043 & Malaria & Malaria \\
\hline Caso_2019 & Malaria & Malaria & Caso_2044 & Malaria & Malaria \\
\hline Caso_2020 & Malaria & Malaria & Caso_2045 & Malaria & Malaria \\
\hline Caso_2021 & Malaria & Malaria & Caso_2046 & Malaria & Malaria \\
\hline Caso_2022 & Malaria & Malaria & Caso_2047 & Sano & Sano \\
\hline Caso_2023 & Malaria & Malaria & Caso_2048 & Malaria & Malaria \\
\hline Caso_2024 & Malaria & Malaria & Caso_2049 & Malaria & Malaria \\
\hline Caso_2025 & Sano & Sano & Caso_2050 & Malaria & Malaria \\
\hline
\end{tabular}

Para el grado de concordancia se utilizó el índice estadístico Kappa (k), cuyo objetivo es proporcionar una medida del grado de acuerdo existente entre dos instrumentos, observadores o jueces al evaluar una serie de sujetos u objetos (Cohen, 1960); es decir, evalúa la concordancia o reproductibilidad de instrumentos de medida cuyo resultado es categórico (2 o más categorías), representando la proporción de acuerdos observados más allá del azar. Así entonces, el valor de este índice es de 0,897 con un $p$-valor $<0,01$, lo cual indica que existe un acuerdo significativamente más alto que el esperado por el azar.

\section{DISCUSIÓN}

Se puede observar que el modelo de sistema propuesto, tiene una similitud en el diagnóstico de cerca del $90 \%$ con el caso en consulta, dentro de una muestra escogida de 50 casos entre los que se encuentran casos de malaria y leishmaniasis.

Lo ideal de este sistema, es que no se crea ningún aplicativo en particular, sino que el sistema propuesto parte del manejo de los softwares libres, y es dentro de ellos en donde se configura las necesidades y requerimientos del sistema mismo.
Finalmente, el resultado es un sistema en el que mediante la creación de una ontología en el software Protégé y el enlazamiento de ésta a través del software jColibri, se puede establecer muy bien un sistema de razonamiento basado en casos, para el diagnóstico de enfermedades como apoyo al médico o profesional de salud y que muy bien se puede portar, simplemente llevando el sistema a accesos remotos, pues ambos softwares son libres.

Asimismo, la confiabilidad de nuestro modelo de sistema RBC, se midió con el índice Kappa de Cohen (1960) con un valor de 0,897 indicando que la concordancia se encuentra en el nivel más alto $(0,81-1,00)$, es decir, el acuerdo entre el modelo RBC propuesto en esta investigación y el diagnóstico médico mostrado en las historias clínicas es "muy bueno". Esto concuerda con el estudio de Herrero (2007), el cual tiene un porcentaje de acierto de $80,76 \%$, bastante cercano nuestra investigación.

\section{REFERENCIAS BIBLIOGRÁFICAS}

Aamodt, A., \& Plaza, E. (1994). Case-based reasoning: Foundational issues, methodological variations, and system approaches. Al communications, 7(1), 3959.156-65. 
Andritsos, P., Jurisica, I., \& Glasgow, J. I. (2014). Case-Based Reasoning for Biomedical Informatics and Medicine. In Springer Handbook of Bio-/Neuroinformatics (pp. 207221). Springer Berlin Heidelberg.

Cohen, J. (1960). A coefficient of agreement for nominal scales. Educational and psychological measurement, 20(1), 37-46.

Cunningham, P. (2009). A taxonomy of similarity mechanisms for case-based reasoning. IEEE Transactions on Knowledge and Data Engineering, 21(11), 1532-1543.

Herrero, J. M. J. (2007). Una aproximación multimodal al diagnóstico temporal mediante razonamiento basado en casos $y$ razonamiento basado en modelos. Aplicaciones en la medicina (Doctoral dissertation, Universidad de Murcia).
Recio-García, J. A., Díaz-Agudo, B., \& GonzálezCalero, P. A. (2014). The COLIBRI platform: tools, features and working examples. In Successful Case-based Reasoning Applications-2, 55-85. Springer Berlin Heidelberg.

Protégé, (2014). Protege, Ontology Editor and Knowledge Acquisition System, Recuperado de http://protege.stanford.edu/.

\section{Correo electrónico:}

miguel_alv26@hotmail.com

Revisión de pares:

Recibido: 05-09-2016

Aceptado: 27-12-2016 